\title{
Zur Bedeutung von akademischer Motivation und Persönlichkeits- merkmalen für den Studienerfolg
}

\author{
Gibt es Unterschiede bei der Berücksichtigung \\ des Migrationshintergrundes?
}

\author{
Mona Höyng [D und Margret Borchert \\ Mercator School of Management, Universität Duisburg-Essen, Duisburg, Deutschland
}

\begin{abstract}
Zusammenfassung: Angesichts der kontrovers diskutierten Bildungsbarrieren und -chancen von Kindern, Jugendlichen und jungen Erwachsenen mit Migrationshintergrund im deutschen Bildungssystem untersucht dieser Beitrag, inwiefern sich die Determinanten des akademischen Erfolgs hinsichtlich des Migrationshintergrundes von Studenten und Studentinnen unterscheiden. Hierbei erfolgt eine empirische Analyse des schulischen Leistungsniveaus, der akademischen Motivation sowie von ausgewählten Persönlichkeitsmerkmalen als Determinanten der akademischen Leistung von Studenten und Studentinnen mit und ohne Migrationshintergrund. Die Stichprobe bestand aus 1854 Studenten und Studentinnen an wirtschaftswissenschaftlichen Fakultäten von sechs verschiedenen deutschen Universitäten, davon wiesen rund ein Drittel der Studenten und Studentinnen einen Migrationshintergrund auf. Die empirischen Befunde zeigen, dass insbesondere die schulische Leistung sowie die Gewissenhaftigkeit die akademische Leistung beider Herkunftsgruppen determinieren. Demgegenüber beeinflussen die Dimensionen der akademischen Motivation weder die akademische Leistung von Studenten und Studentinnen ohne Migrationshintergrund noch diejenige der Studenten und Studentinnen mit Migrationshintergrund.
\end{abstract}

Schlüsselwörter: Bildungsungleichheiten, Migration, Akademische Leistung, Akademische Motivation, Big-Five Persönlichkeitsmerkmale

The role of academic motivation and personality traits in determining academic performance: Are there any differences when considering the migration background?

\begin{abstract}
Based on the controversial debate regarding the educational barriers and opportunities of children, adolescents, and young adults with a migration background in the German education system this article examines to what extent the determinants of students' academic performance differ in terms of their migration background. Hereby, we empirically investigate the level of performance at school, the academic motivation and selected personality traits as determinants of the academic performance of students with and without a migration background. The sample included 1854 students at economic faculties of six different German universities. Thereby, one-third of the students had a migration background. The empirical results show that in particular the level of performance at school as well as the level of conscientiousness determine the academic performance among both groups of origin. In contrast, the dimensions of academic motivation neither influence the academic performance of students without a migration background, not that of students with a migration background.
\end{abstract}

Keywords: Educational inequalities, migration, academic performance, academic motivation, big five personality traits

Bildungsungleichheiten und Probleme mangelnder Bildungserfolge zwischen Kindern, Jugendlichen und jungen Erwachsenen mit und ohne Migrationshintergrund ${ }^{1}$ sind in den letzten Jahren verstärkt in den Fokus der Bildungs- und Hochschulforschung gerückt (z.B. Kristen, 2016). Hier wurden Ursachen von Bildungsungleichheiten und die Probleme mangelnder Bildungserfolge bei bestimmten Migrantengruppen und in spezifischen Bildungsetappen kontrovers diskutiert (z.B. Diehl, Hunkler \& Kristen, 2016; Raiser, 2007; Tepecik, 2011). Neben einer einseitigen und

Für die Einstufung als „Person mit Migrationshintergrund“ wird der Definition des Statistischen Bundesamtes gefolgt. Demzufolge liegt ein Migrationsstatus vor, sofern das Individuum selbst oder mindestens ein Elternteil nicht in Deutschland geboren ist und nicht die deutsche Staatsbürgerschaft von Geburt an besitzt (Statistisches Bundesamt, 2019). 
pauschalen „defizitorientierten“ Darstellung des mangelnden Bildungserfolges von Migrantinnen und Migranten², thematisiert Tepecik (2011) eine „ressourcenorientierte Perspektive“, in welcher „bildungserfolgreiche“ Individuen mit Migrationshintergrund im Fokus einzelner Bildungsetappen stehen. In der primären und sekundären Ausbildung werden die schlechteren schulischen Leistungen von Schulkindern mit Migrationshintergrund im Vergleich zu ihren deutschen Mitschülern, u. a. auf sprachliche Probleme und ethnisch bedingte Unterschiede ${ }^{3}$ zurückgeführt (z. B. Kristen, 2016; OECD, 2016; Tepecik, 2011). Im Bereich der tertiären Bildung existieren jedoch bislang inkonsistente Befunde zu den Determinanten von Leistungsunterschieden zwischen Studienberechtigten mit und ohne Migrationshintergrund. Insbesondere mangelt es in diesem Kontext an empirischen Befunden. Da der Bildungserfolg von Studienberechtigten mit Migrationshintergrund jedoch insbesondere im tertiären Bildungsbereich für eine erfolgreiche Eingliederung in den Arbeitsmarkt (Diehl, Hunkler \& Kristen, 2016) sowie die Integration in die Gesellschaft von großer Bedeutung ist (Becker, 2011), bedarf es einer tiefergehenden Analyse. Auf Basis vergangener Studien ist von keiner grundsätzlichen Benachteiligung von Studenten mit Migrationshintergrund auszugehen (z.B. Kristen, 2014). Da der Bereich der tertiären Bildung als späte und hochselektive Phase im Bildungsverlauf gilt, sind Studenten mit Migrationshintergrund als positiv selektierte Gruppe einzustufen (Kristen, 2014, 2016; Tepecik, 2011). In diesem Kontext zeigen Befunde von Kristen (2014), dass Studenten mit Migrationshintergrund - bei Annahme einer gleichen Leistungsausgangslage - eine höhere Studierneigung im Vergleich zu Studienberechtigten ohne Zuwanderungshintergrund aufweisen (Kristen, 2014). Allerdings ist festzustellen, dass sich Studienberechtigte mit Migrationshintergrund aufgrund ihrer schulischen Leistungen in einer schlechteren Ausgangslage befinden (Kristen, 2014). Ferner wurde die schulische Leistung als relevanter Prädikator des Studienerfolgs von Studenten mit Migrationshintergrund in Deutschland identifiziert (Brändle \& Lengfeld, 2015) und wird somit zur Erklärung von Unterschieden in der akademischen Leistung (AL) von Studenten mit und ohne Migrationshintergrund herangezogen. Als weitere Ursache für akademische Leistungsunterschiede zwischen diesen beiden Gruppen wird zum einen im Rahmen der institutionellen Diskriminierung die geringe Öffnung von Institutionen für Migranten in der Aufnahmegesellschaft thematisiert (Moffitt, Juang \& Syed, 2019; Sprung, 2017). Zum anderen stellen soziostrukturelle Faktoren, wie z.B. unterschiedliche Bildungshintergründe, soziale Herkünfte und familiäre Kulturen, zentrale Determinanten von Unterschieden in der AL zwischen Studenten mit und ohne Migrationshintergrund dar (z.B. Bülow-Schramm, 2016; Dekker \& Fischer, 2008; Ebert \& Heublein, 2017). Ferner werden familiäre Ressourcen, wie z.B. die elterliche oder finanzielle Unterstützung, intensiv in der tertiären Bildungsforschung diskutiert (Diehl, Hunkler \& Kristen, 2016). Daneben determinieren Faktoren, wie beispielsweise die Bildungs- oder Lernmotivation oder die individuelle Persönlichkeit der Studenten, den Bildungserfolg von Individuen (Diehl, Hunkler \& Kristen, 2016; Kristen, 2016; Röbken \& Mertens, 2013). In diesem Zusammenhang stellt Kristen (2016) heraus, dass Studenten mit Migrationshintergrund eine höhere Motivation für ein Studium, d.h. eine höhere akademische Motivation, aufweisen. Ob und inwiefern die erhöhte akademische Motivation jedoch auch die akademische Leistung der Studenten mit Migrationshintergrund positiv beeinflusst, ist unklar. Aufgrund fehlender multivariater Auswertungen adressiert das erste Forschungsziel dieses Beitrages die bislang fehlende empirische Untersuchung des Zusammenhanges zwischen der akademischen Motivation und der AL unter Berücksichtigung des Migrationshintergrundes. Ferner ist zu berücksichtigen, dass die Persönlichkeit als individueller Faktor in einem Zusammenhang zur AL steht, wobei die Big-Five Persönlichkeitsmerkmale die bisherige Forschung in diesem Bereich dominieren (Higgins, Peterson, Pihl \& Lee, 2007; Röbken \& Mertens, 2013). Während die Gewissenhaftigkeit als stärkster positiver und der Neurotizismus als stärkster negativer Prädiktor der AL identifiziert werden, erwiesen sich die übrigen drei Persönlichkeitsmerkmale als nicht signifikante Determinanten der AL (Hillebrecht, 2019). Da bislang jedoch keine empirischen Befunde hinsichtlich der Differenzierung der Persönlichkeitsmerkmale nach der ethnisch bedingten Herkunft von Studenten existieren, adressiert das zweite Forschungsziel die empirische Untersuchung der Zusammenhänge zwischen der Gewissenhaftigkeit sowie dem Neurotizismus als Persönlichkeitsmerkmale und der AL unter Berücksichtigung des Migrationshintergrundes.

Zur Analyse der Determinanten der AL zwischen den Herkunftsgruppen werden die Zusammenhänge zwischen der akademischen Motivation sowie zwischen der Gewissenhaftigkeit bzw. dem Neurotizismus und der AL von Studenten mit und ohne Migrationshintergrund nachfolgend hergeleitet.

\footnotetext{
Nachfolgend wird ausschließlich aus Gründen der besseren Lesbarkeit der generische Maskulin verwendet. Maskuline Bezeichnungen schließen stets das weibliche Geschlecht ein.

In Anlehnung an Kristen (2016) umfasst diese Bezeichnung synonyme Begrifflichkeiten, die sich auf den Zuwanderungshintergrund der Studenten beziehen. Die Bezeichnung erfasst insgesamt Unterschiede im Bildungserfolg (z. B. migrationsspezifische Bildungsungleichheiten) zwischen verschiedenen Herkunftsgruppen und der Aufnahmegesellschaft.
} 


\section{Zusammenhang zwischen akademischer Motivation und akademischer Leistung}

Aufbauend auf der Selbstbestimmungstheorie von Deci und Ryan ist die akademische Motivation entlang eines Selbstbestimmungskontinuums in drei Dimensionen, die extrinsische und intrinsische Motivation sowie die Amotivation zu differenzieren (Ryan \& Deci, 2000a, 2000b). Das Kontinuum spiegelt dabei den Grad des selbstbestimmten Verhaltens wider und beginnt am unteren Ende mit der Amotivation (AM), die den geringsten Grad an Selbstbestimmung abbildet. Von dort aus reicht das Kontinuum über die extrinsische Motivation (EM) bis hin zur intrinsischen Motivation (IM), welche das andere Ende des Kontinuums und damit den höchsten Grad an Selbstbestimmung darstellt (Ryan \& Deci, 2000a, 2000b). Ferner ist die EM entlang des Selbstbestimmungskontinuums in drei weitere Subdimensionen zu differenzieren (z.B. Deci, Vallerand, Pelletier \& Ryan, 1991). Von niedrigeren bis höheren Graden an selbstbestimmtem (bzw. kontrolliertem) Verhalten untergliedern sich diese in die externale, introjizierte und die identifizierte EM (Deci et al., 1991). Von der ursprünglich vorgeschlagenen vierten Dimension, der integrierten EM, ist aufgrund zu starker Überschneidungen mit der IM hinsichtlich der autonomen Selbstregulierung Abstand zu nehmen (Deci et al., 1991). Um den Zusammenhang zwischen der akademischen Motivation und der AL herzuleiten, wird das Überblicksmodell des motivierten Handelns von Heckhausen \& Heckhausen (2018) herangezogen. Demnach ergibt sich die gegenwärtige Motivation eines Individuums, ein spezifisches Ziel zu verfolgen, aus der Interaktion zwischen situativen Anreizen und persönlichen Gegebenheiten (Heckhausen \& Heckhausen, 2018). Die Ausprägung der Motivation impliziert schließlich unterschiedliche Handlungsweisen und beeinflusst somit die Leistung. Bisherige empirische Befunde bestätigen, dass eine steigende Motivation mit einer Verbesserung der AL von Studenten einhergeht (z.B. Richardson, Abraham \& Bond, 2012). Hierbei wurde die akademische Motivation als Einflussfaktor der AL vorwiegend in der angloamerikanischen Literatur analysiert (Komarraju, Karau \& Schmeck, 2009). Dahingehend ist bislang unklar, inwiefern sich dieser Wirkungszusammenhang zwischen den einzelnen Subdimensionen der akademischen Motivation und der AL sowie darüber hinaus zwischen Studenten mit und ohne Migrationshintergrund differenziert.

Hinsichtlich der EM wird grundsätzlich erfasst, dass Studenten nicht zwangsläufig selbstbestimmt handeln, d.h. nicht aus persönlichen Motiven heraus. Vielmehr steigern Studenten ihre Leistungen durch den Einfluss von äußeren Faktoren, wie beispielsweise die Belohnung und Akzeptanz durch Gleichaltrige bzw. durch die Eltern. In Bezug auf den Migrationshintergrund stellen Röbken \& Mertens (2013) heraus, dass Individuen mit Migrationshintergrund im Vergleich zu Individuen ohne Migrationshintergrund stärker extrinsisch und intrinsisch für ein Studium motiviert sind (Röbken \& Mertens, 2013). Als extrinsische Anreize sind beispielsweise die angestrebte gesellschaftliche Integration, die Anerkennung durch Dritte oder der Abschluss einer Klausur mit einer überdurchschnittlichen Note interpretierbar, welche die AL der Studenten positiv beeinflussen (Tjaden \& Hunkler, 2017). Die Zusammenhänge zwischen den Subdimensionen der EM und der AL lassen sich für Studenten mit und ohne Migrationshintergrund wie folgt differenzieren.

Die externale EM wird durch die zu erwartenden Belohnungen bzw. drohenden Bestrafungen reguliert und ist daher nicht selbstbestimmt (Deci et al., 1991; Vallerand, Pelletier, Blais, Briere, Senecal \& Vallieres, 1992). Es ist zu erwarten, dass das Handeln von Studenten mit Migrationshintergrund stärker durch die zu erwartenden Belohnungen, im Sinne der externalen EM, beeinflusst wird als unter Studenten ohne Migrationshintergrund. So umfassen ausgehend von der Immigrant-OptimismHypothesis die erwarteten Belohnungen von Studenten mit Migrationshintergrund den Drang, durch Bildung einen sozialen Aufstieg zu erreichen sowie den Wunsch nach kultureller sowie sozialer Assimilation an die Aufnahmegesellschaft und Integration in das Statussystem dieser Gesellschaft (Kao \& Tienda, 1995; Kristen, Reimer \& Kogan, 2008; Nauck, 2001; Tjaden \& Hunkler, 2017). Gleichzeitig sind für Studenten mit Migrationshintergrund der Erwerb von Wissen und Kompetenzen notwendige Voraussetzungen für den künftigen Erfolg am Arbeitsmarkt in der Aufnahmegesellschaft (Becker, 2011). Aus diesem Grund ist zu erwarten, dass sich das Verhalten und die AL der Studenten stark an den externen Rahmenbedingungen und den zu erwartenden Belohnungen, welche als externale EM definiert werden, orientieren. Im Einklang mit dem Überblicksmodell des motivierten Handelns verstärkt die Motivationsausprägung daher die AL dieser Gruppe. Dieser vermutete Wirkungszusammenhang konnte in vergangenen empirischen Befunden bestätigt werden (z.B. Areepattamannil \& Freeman, 2008). Da diese extrinsischen Anreize für Studenten ohne Migrationshintergrund in dieser Form nicht vorliegen, ist für diese Gruppe von einem schwächer ausgeprägten $\mathrm{Zu}-$ sammenhang zwischen der externalen EM und der AL auszugehen. Demzufolge ist folgende Hypothese anzunehmen:

H1a: Der positive Zusammenhang zwischen der externalen EM und der AL ist für Studenten mit Migrationshintergrund stärker als für Studenten ohne Migrationshintergrund. 
Die introjizierte EM ist durch externe Faktoren geprägt und wird in einem abgegrenzten Rahmen von Werten und Erwartungen, der mit externer Kontrolle und Zwängen einhergeht, realisiert. Somit ermöglicht die introjizierte EM kein selbstbestimmtes Verhalten (Deci et al., 1991; Vallerand et al., 1992). Bisherige empirische Befunde zeigen einen positiv signifikanten Zusammenhang zwischen der introjizierten EM und der AL (Sartawi, Alsawaie, Dodeen, Tibi \& Alghazo, 2012). Im Folgenden wird angenommen, dass das Verhalten und somit die AL von Studenten mit Migrationshintergrund stärker durch die Verinnerlichung von externen Anreizen reguliert wird als unter Studenten ohne Migrationshintergrund. Diese Annahme ist ausgehend von der Immigrant-OptimismHypothesis damit zu begründen, dass hohe Bildungsabschlüsse der Kinder als eine Möglichkeit für die Eltern angesehen werden, die eigenen Probleme in Bezug auf die Integration in und Anpassung an die Aufnahmegesellschaft zu lösen. So konnte nachgewiesen werden, dass der erwünschte soziale Aufstieg und die Erwartungen der Eltern extrinsische Anreize für Studenten mit Migrationshintergrund darstellen, welche die AL der Studenten positiv beeinflussen (Tjaden \& Hunkler, 2017). Demnach wird vermutet, dass die Werte und Ziele von Studenten mit Migrationshintergrund zunächst extern durch ihre Eltern an die Studenten weitergegeben werden und diese dann von den Studenten verinnerlicht und akzeptiert werden (Kao \& Tienda, 1995; Tjaden \& Hunkler, 2017). Ferner ist zu erwarten, dass Studenten mit Migrationshintergrund u.a. die Bildungsaspirationen, die Werte und Einstellungen ihrer Eltern, wie z. B. einen ausgeprägten Optimismus sowie Ehrgeiz verinnerlichen und auf diese Weise ihre AL verbessern (Tepecik, 2011; Tjaden \& Hunkler, 2017). Folglich ist ein positiver Zusammenhang zwischen der introjizierten EM und der AL für Studenten mit Migrationshintergrund zu erwarten. Dieser Zusammenhang kann ferner ausgehend vom Value-of-Children-Ansatz (VOC-Ansatz) hergeleitet werden. Demnach sind die Eltern-KindBeziehungen in Deutschland tendenziell eher psychologisch-affektiv geprägt, während in migrierten Familien häufiger ökonomisch-utilitaristische Beziehungen ${ }^{4}$ vorliegen (Carnein \& Baykara-Krumme, 2013; Olbermann, 2003). Letztere zeichnen sich durch eine reziproke Erwartungshaltung, die migrierte Eltern gegenüber ihren Kindern haben, aus. Diese entsteht aus der Verantwortlichkeit der Eltern für die Erziehung ihrer Kinder sowie der im Gegenzug im Alter notwendigen materiellen und immateriellen Versorgung der Eltern durch ihre Kinder (z.B. Nauck, 2000). Aufgrund den daraus resultierenden normativen Erwartungen ist für Studenten mit Migrationshintergrund eine höhere introjizierte EM zu erwarten. Dies ist damit zu begründen, dass die Verhaltensweisen der Studenten weiterhin maßgeblich durch externe Faktoren getrieben werden, wobei die Erwartungen und Anforderungen der Eltern teilweise bereits verinnerlicht werden. Im Einklang mit dem Überblicksmodell des motivierten Handelns ist anzunehmen, dass die ausgeprägte introjizierte EM dieser Studenten zu einer Verbesserung der AL führt (Heckhausen \& Heckhausen, 2018). Demgegenüber basieren psychologisch-affektiv geprägte Beziehungen in Familien ohne Migrationshintergrund auf einer gesteigerten Gruppenbindung sowie einer emotionalen Stimulation der Eltern durch den Umgang mit ihren Kindern und implizieren keine gesteigerten extrinsischen Anreize. Folglich ist auch keine Verbesserung der AL zu erwarten. Daraus ergibt sich die folgende Hypothese:

H1b: Der positive Zusammenhang zwischen der introjizierten EM und der AL ist für Studenten mit Migrationshintergrund stärker als für Studenten ohne Migrationshintergrund.

Im Rahmen der identifizierten EM verinnerlichen Studenten fremde Ziele als ihre eigenen und assimilieren die eigenen Werte und Erwartungen an ein extern umgebendes Wertesystem. Die Studenten haben das Gefühl eigenständig und selbstbestimmt zu handeln. Die bisherigen Befunde bestätigen einen positiven Zusammenhang zwischen der identifizierten EM und der AL (z.B. Ryan \& Deci, 2000a). Im Vergleich zu Studenten ohne Migrationshintergrund ist daher für Studenten mit Migrationshintergrund ein stärkerer positiver Zusammenhang zwischen der identifizierten EM und der AL anzunehmen. Dies liegt daran, dass Studenten mit Migrationshintergrund die Ziele ihrer Eltern, wie z.B. eine gesellschaftliche Integration, vollständig als eigene Ziele verinnerlichen. Dadurch entsteht eine erhöhte Motivationsausprägung, die angelehnt an das Überblicksmodell des motivierten Handelns eine Verbesserung der AL vermuten lässt. Da ausgehend vom VOC-Ansatz diese zusätzlichen extrinsischen Anreize für Studenten ohne Migrationshintergrund in der Form nicht vorliegen, ist insgesamt davon auszugehen, dass der Zusammenhang für diese Gruppe von Studenten schwächer ausgeprägt ist. Die zu untersuchende Hypothese lautet wie folgt:

H1c: Der positive Zusammenhang zwischen der identifizierten EM und der AL ist für Studenten mit Migrationshintergrund stärker als für Studenten ohne Migrationshintergrund.

Demgegenüber beinhaltet die intrinsische Motivation (IM) Verhaltensweisen, die innerhalb der Person selbstbestimmt, aus reiner Neugier und eigenem Willen entstehen (z.B. Deci et al., 1991; Röbken \& Mertens, 2013). Die IM

Die ökonomisch-utilitaristische Ausprägung von Familienbeziehungen wurde insbesondere in türkischen Familien nachgewiesen (z.B. Nauck, 2000). 
wirkt sich über die Motivation etwas zu erreichen positiv auf die AL von Studenten aus (Goodman et al., 2011; Turner, Chandler \& Heffer, 2009; Vansteenkiste, Lens \& Deci, 2006). Studenten mit Migrationshintergrund wurden auf Basis der bisherigen Befunde als positiv selektierte Gruppe identifiziert, welcher ein erhöhter Optimismus und Ehrgeiz unterstellt wird (z.B. Kristen, 2014; Tepecik, 2011; Tjaden \& Hunkler, 2017). Folglich ist für diese Gruppe von Studenten ein ausgeprägter Grad an selbstbestimmtem Verhalten im Sinne der IM zu erwarten, welcher im Vergleich zu Studenten ohne Migrationshintergrund zu einer stärkeren Verbesserung der AL führt. Gleichzeitig ist angelehnt an den VOC-Ansatz zu vermuten, dass die familiär bedingt vermehrt auftretenden extrinsischen Anreize von Studenten mit Migrationshintergrund den Zusammenhang zwischen der IM und der AL verstärken. Aufgrund der vermuteten Verinnerlichung von elterlichen Werten und Zielen durch die Kinder ist ein Gleichlauf ihrer Interessen zu erwarten. Daraus resultiert eine Steigerung der IM, welche im Einklang mit dem Überblicksmodell des motivierten Handelns eine Verbesserung der AL vermuten lässt (Heckhausen \& Heckhausen, 2018) und letztlich einen stärkeren Zusammenhang zwischen der IM und der AL unter Studenten mit Migrationshintergrund im Vergleich zu Studenten ohne Migrationshintergrund erklären würde. Unter zusätzlicher Berücksichtigung von institutioneller Diskriminierung, der Studenten mit Migrationshintergrund nachweislich ausgesetzt sind (Fereidooni, 2012), ist jedoch eher von einem deutlich abgeschwächten positiven Zusammenhang zwischen der IM und der AL für Studenten mit Migrationshintergrund auszugehen. Hierbei bezieht sich die institutionelle Diskriminierung auf dauerhafte Benachteiligungen von sozialen Gruppen (Hasse \& Schmidt, 2012). Dazu gehören eine Vielzahl von Faktoren, wie z.B. ein diskriminierendes und stereotypisches Verständnis von Nicht-Migranten hinsichtlich der Zugehörigkeit von Migranten zur Universität als Bildungseinrichtung (Moffitt, Juang \& Syed, 2019; Sprung, 2017). Zusätzlich ist im Rahmen der ,defizitorientierten Perspektive" zu vermuten, dass die vielfach nachgewiesenen Diskriminierungen von Migranten (Moffitt, Juang \& Syed, 2019; Tepecik, 2011) die Gefühle von Studenten mit Migrationshintergrund negativ dominieren. Eine resultierende Reduktion der Motivation lässt in Anlehnung an das Überblicksmodell des motivierten Handelns eine Abschwächung des positiven Zusammenhangs zwischen der IM und der AL von Studenten mit Migrationshintergrund im Vergleich zu Studenten ohne Migrationshintergrund vermuten. Insgesamt wird daher für Studenten mit Migrationshintergrund ein Verdrängungseffekt angenommen, welcher die IM angesichts ausgeprägter extrinsischer Faktoren reduziert und daher den positiven Zusammenhang zwischen der IM und der AL abschwächt. Da diese extrin- sischen Anreize für Studenten ohne Migrationshintergrund, wie oben dargestellt, nicht vorhanden sind und Diskriminierung in dieser Form nicht vorliegt, ist für diese Gruppe von Studenten zunächst nicht von einem Verdrängungseffekt auszugehen. Daher wird folgende Hypothese angenommen:

H2: Der positive Zusammenhang zwischen der IM und der $A L$ ist für Studenten mit Migrationshintergrund schwächer als für Studenten ohne Migrationshintergrund.

Die Amotivation (AM) als dritte Ausprägung der akademischen Motivation bezieht sich auf nicht-intentionale Verhaltensweisen, welche kein spezifisches Ziel verfolgen (z.B. Vallerand et al., 1992). Zum Zusammenhang zwischen der AM und der AL von Studenten belegen die vorhandenen empirischen Befunde plausible negative Zusammenhänge (z. B. Turner, Chandler \& Heffer, 2009). Im Einklang mit dem Überblicksmodell des motivierten Handelns hängen dementsprechend die fehlende Motivation sowie das als gering wahrgenommene Potential im eigenen Handeln mit einer schlechteren AL im Vergleich $\mathrm{zu}$ motivierten Studenten zusammen (Heckhausen \& Heckhausen, 2018; Vallerand et al., 1992). Unklar ist jedoch, inwiefern sich dieser Zusammenhang zwischen Studenten mit und ohne Migrationshintergrund unterscheidet. Zum einen wurden Studenten mit Migrationshintergrund als positiv selektierte Gruppe identifiziert (z.B. Tepecik, 2011), die unter Bezugnahme des VOCAnsatzes und der Immigrant-Optimism-Hypothesis ausgeprägte extrinsische und intrinsische Anreize wahrnehmen. Daher ist zunächst zu vermuten, dass sich die AM unter Studenten mit Migrationshintergrund im Vergleich zu Studenten ohne Migrationshintergrund schwächer negativ auf ihre AL auswirkt. Jedoch sind die schlechteren schulischen Leistungen von Studenten mit Migrationshintergrund sowie die im Studium relevanten herkunftsbedingten Benachteiligungen (Kristen, 2016) ebenfalls zu berücksichtigen. Im Vergleich zu Studenten ohne Migrationshintergrund machen sich diese negativen Erfahrungen von Studenten mit Migrationshintergrund vermutlich bemerkbar und wirken sich negativ auf den Zusammenhang zwischen der AM und der AL aus. Verstärkt wird diese Vermutung durch den Aspekt der institutionellen Diskriminierung, die den Bildungsaufstieg der Studenten mit Migrationshintergrund hemmt (Moffitt, Juang \& Syed, 2019; Sprung, 2017). Die geringe Motivausprägung dieser Studenten führt schließlich zu einer Verschlechterung ihrer AL (Heckhausen \& Heckhausen, 2018). Die Vermutung eines stärkeren negativen Zusammenhangs zwischen der AM und der AL für Studenten mit Migrationshintergrund im Vergleich zu Studenten ohne Migrationshintergrund ist konsistent zu den zuvor getroffenen Hypothesen. Aufgrund der vermuteten besonders starken Bedeutung von extrinsischen Anrei- 
zen und dem dadurch geringen Autonomieempfinden gegenüber den Eltern wird für Studenten mit Migrationshintergrund viel Raum für AM gesehen, welcher zu einer stärkeren Verschlechterung der AL führt (Ryan \& Deci, 2000a; Vallerand et al., 1992). Im Vergleich dazu liegen unter Studenten ohne Migrationshintergrund bessere Startbedingungen für das akademische Studium, keine herkunftsbedingten Nachteile sowie wenig Raum für institutionelle Diskriminierung vor. Insgesamt ist folglich mit Hypothese 3 von folgendem Zusammenhang auszugehen:

H3: Der negative Zusammenhang zwischen der AM und der AL ist für Studenten mit Migrationshintergrund stärker als für Studenten ohne Migrationshintergrund.

\section{Zusammenhang zwischen Persön- lichkeit und akademischer Leistung}

Als zweiter relevanter Parameter wird die individuelle Persönlichkeit im Zusammenhang mit der AL von Studenten mit und ohne Migrationshintergrund untersucht. Im Einklang mit dem Überblicksmodell des motivierten Handelns werden Persönlichkeitsmerkmale als individuelle Aspekte der Persönlichkeit und zeitstabile Eigenschaften von Studenten betrachtet (Costa \& McCrae, 1992; Heckhausen \& Heckhausen, 2018; McCrae et al., 2000). In diesem Kontext wurden die Persönlichkeitsmerkmale von Migranten als Indikator für die wahrgenommene Diskriminierung identifiziert, welche den Bildungserfolg dieser Gruppe negativ beeinflussen (z. B. El-Mafaalani, Waleciak \& Weitzel, 2017). Nachfolgend wird die Persönlichkeit der Studenten mithilfe der Big-Five Persönlichkeitsmerkmale Neurotizismus, Extraversion, Offenheit für Erfahrungen, Verträglichkeit und Gewissenhaftigkeit erfasst (Costa \& McCrae, 1992). In der bisherigen Forschung zum direkten Zusammenhang zwischen den Persönlichkeitsmerkmalen und der AL stellen die Gewissenhaftigkeit den stärksten positiven und der Neurotizismus den stärksten negativen Parameter dar (Higgins et al., 2007; Hillebrecht, 2019; Komarraju, Karau \& Schmeck, 2009; Wach, Karbach, Ruffing, Brünken \& Spinath, 2016). Die übrigen drei Persönlichkeitsmerkmale weisen keinen wissenschaftlich fundiert begründbaren Zusammenhang zur AL auf und werden hier daher nicht weiter betrachtet (Hillebrecht, 2019). Insofern konzentrieren sich die nachfolgenden Ausführungen auf die Persönlichkeitsmerkmale Gewissenhaftigkeit und Neurotizismus. Da es dahingehend bislang an einer Analyse mangelt, welche die Persönlichkeitsmerkmale zwischen Studenten mit und ohne Migrationshintergrund differenziert (Cano et al., 2012), wird dies im Folgenden fokussiert.
Gewissenhafte Studenten weisen grundsätzlich ein hohes Maß an Disziplin auf und werden als ehrgeizig, belastbar und zuverlässig charakterisiert (z.B. Poropat, 2009). Durch den ausgeprägten Willen, etwas zu erreichen, erzielen diese Studenten im Durchschnitt bessere Leistungen im Vergleich zu nicht gewissenhaften Studenten (Chamorro-Premuzic \& Furnham, 2003; Komarraju, Karau \& Schmeck, 2009; Poropat, 2009). Unter Berücksichtigung des Migrationshintergrundes belegen Cano et al. (2012) im schulischen Kontext, dass gewissenhafte Schüler mit Migrationshintergrund, welche zudem gewillt sind, sich an die Kultur der Aufnahmegesellschaft anzupassen, eine höhere Erwartung an die eigene Bildung haben. Im tertiären Bildungsbereich gelten Studenten mit Migrationshintergrund als positiv selektierte Gruppe, die die Kompetenzen ihrer Eltern übertreffen und neues Wissen erlernen wollen (z. B. Raiser, 2007). Zusätzlich ist zu erwarten, dass die Sorgfalt und Zielorientierung sowie das Pflichtbewusstsein von Studenten durch den Einfluss der Eltern verstärkt werden. Ausgehend von der Immigrant-Optimism-Hypothesis ist zu vermuten, dass der erhöhte Optimismus und Ehrgeiz unter migrierten Eltern u.a. eine erhöhte Zielorientierung und ein gesteigertes Pflichtbewusstsein mit sich bringen. Die ausgeprägte Gewissenhaftigkeit wird von den Eltern an die Kinder weitergegeben und impliziert schließlich eine Verbesserung der AL unter Studenten mit Migrationshintergrund (z.B. Tjaden \& Hunkler, 2017). Im Vergleich dazu liegen unter Studenten ohne Migrationshintergrund diese Aspekte, die zu einer Verstärkung des positiven Zusammenhangs zwischen der Gewissenhaftigkeit und der AL führen, in der Form nicht vor. Auf Basis dieser Überlegungen leitet sich die nachfolgende Hypothese ab:

H4: Der positive Zusammenhang zwischen der Gewissenhaftigkeit und der AL ist für Studenten mit Migrationshintergrund stärker als für Studenten ohne Migrationshintergrund.

Demgegenüber geht der Neurotizismus mit einer erhöhten Angst zu versagen, einer emotionalen Instabilität sowie einer reduzierten intellektuellen Neugier einher. Deshalb wird der Neurotizismus mit einer Verschlechterung der AL assoziiert (Chamorro-Premuzic \& Furnham, 2003; Komarraju, Karau \& Schmeck, 2009). Bislang mangelt es jedoch an empirischen Befunden, die diesen negativen Zusammenhang zwischen dem Neurotizismus und der AL hinsichtlich des Migrationshintergrundes von Studenten differenzieren. Relevant erscheint hier der Hinweis von Jancz (2000), der davon ausgeht, dass neurotische Studenten mit Migrationshintergrund verstärkt Probleme haben, sich an die unbekannte Aufnahmegesellschaft anzupassen. Ferner wurde für Studenten mit Migrationshintergrund eine fehlende Vertrautheit mit dem deutschen Hochschulsystem nachgewiesen (Kristen, 2016). Darüber hinaus bestätigen empirische Befunde eine ver- 
stärkte Unsicherheit von neurotischen Studenten, die einer ethnischen Minderheit zugehören und keine Machtposition innehalten (El-Mafaalani, Waleciak \& Weitzel, 2017). Insofern ist zu vermuten, dass Studenten mit Migrationshintergrund mit herkunftsbedingten Erschwernissen im tertiären Bildungssystem, für sie undurchsichtigen Studienbedingungen und ungewissen Sozialisationserfolgen im Bereich der gesellschaftlichen Integration konfrontiert sind. Damit verbunden sind Ängste sowie Sorgen vor Rückschlägen, die aufgrund von ökonomisch-utilitaristischen Familienbeziehungen eher noch verschärft werden. Dementsprechend ist nachfolgend von einem stärkeren negativen Zusammenhang zwischen dem Neurotizismus und der AL von Studenten mit Migrationshintergrund im Vergleich zu Studenten ohne Migrationshintergrund auszugehen:

H5: Der negative Zusammenhang zwischen dem Neurotizismus und der AL ist für Studenten mit Migrationshintergrund stärker als für Studenten ohne Migrationshintergrund.

\section{Methode}

\section{Design und Stichprobe}

Da für leistungsbezogene Analysen von Studenten mit Migrationshintergrund zumeist nur geringe Fallzahlen vorliegen (z. B. Kristen, 2016), wurde eine eigene Datenerhebung an wirtschaftswissenschaftlichen Fakultäten von sechs verschiedenen Universitäten in Nordrhein-Westfalen durchgeführt. Im Vergleich zu Datenbanken wie NEPS ermöglichte die eigene Datenerhebung zudem den Einsatz der deutschen Version der AMS (Koch, Schatz \& Borchert (in Druck)). Die Zielgruppe der Erhebungen bildeten Studenten an den Universitäten in Bochum, Dortmund, Duisburg-Essen, Düsseldorf, Köln und Wuppertal, die im Mai 2017 in verschiedenen Vorlesungen befragt wurden. Zur Erweiterung der Stichprobe wurde die Datenerhebung im Mai 2018 wiederholt, wobei die unterschiedlichen Zeitpunkte keine Längsschnittanalysen ermöglichen. Insgesamt wurden 2025 Fragebögen generiert. Nach Ausschluss von Fragebögen, in denen Studenten angaben, bereits teilgenommen zu haben $(N=10)$, und der Bereinigung von nicht vollständig ausgefüllten Fragebögen $(N=161)$, verblieben insgesamt 1854 Fälle im Datensatz. Im bereinigten Datensatz weist etwa ein Drittel der Studenten $(N=621)$ einen Migrationshintergrund auf, während zwei Drittel der Studenten $(N=1233)$ keinen Migrationshintergrund haben. Insgesamt entstammen 43,30\% der Studenten einem akademischen Elternhaus. Unter den Studenten mit Migrationshintergrund sind etwas mehr als ein Drittel $(35,10 \%)$ in einem akademischen El- ternhaus aufgewachsen, während unter den Studenten ohne Migrationshintergrund sogar 47,45\% aus einem akademischen Elternhaus kommen. In der gesamten Stichprobe ist knapp die Hälfte der Befragten männlich (49,70 \%) bzw. weiblich (50,30\%). Das Durchschnittsalter der Befragten beträgt rund 22 Jahre $(S D=2,72)$.

\section{Messinstrumente}

Die $A L$ stellt die abhängige Variable dar und wird als Indikator für den akademischen Erfolg über die Durchschnittsnote im Studium erfasst (Richardson, Abraham \& Bond, 2012). Die Kodierung der Durchschnittsnote erfolgt derart, dass anders als im deutschen Notensystem eine Erhöhung der Note eine Verbesserung der Leistung darstellt (Koch, Schatz \& Borchert (in Druck)).

Als zentrale unabhängige Variable wird der Migrationshintergrund der Studenten erfasst. Für die Kategorie „Studenten mit Migrationshintergrund" gibt es bisher weder eine einheitliche Definition noch eine einheitliche Operationalisierung (Bienfait, 2015). Obwohl der Fokus auf dem Zuwanderungshintergrund der Studenten liegt, können verschiedene Aspekte wie u. a. die eigene Staatsangehörigkeit, das Geburtsland des Studenten oder seiner Eltern oder die in der Familie gesprochene Sprache einen Indikator für den Migrationshintergrund darstellen (Bienfait, 2015; Igler, Ohle-Peters \& McElvany, 2019; Kristen, 2016). Im vorliegenden Beitrag wird die Definition des Statistischen Bundesamtes verwendet. Demnach erfolgt die Einstufung als „Student mit Migrationshintergrund“, wenn der Student selbst oder mindestens ein Elternteil nicht in Deutschland geboren ist und nicht die deutsche Staatsbürgerschaft von Geburt an besitzt (Statistisches Bundesamt, 2019).

Ferner wird die schulische Leistung der Studenten als unabhängige Variable analog zur AL über die Durchschnittsnote im Abitur erfasst. Um die Interpretation der schulischen Leistung zu erleichtern, werden die Ausprägungen dieser Variable umgepolt.

Als weitere unabhängige Variable erfolgt die Messung der akademischen Motivation der Studenten. Diese wurde in Anlehnung an die Academic Motivation Scale (AMS) von Vallerand et al. (1992) mit einer deutschen Übersetzung von Koch, Schatz \& Borchert (in Druck) mithilfe von 28 Items gemessen und in drei Dimensionen differenziert. Die Antworten wurden auf einer fünfstufigen Likert-Skala ( 1 = „stimmt gar nicht“ bis $5=$,stimmt völlig“") gegeben. Über die Eingangsfrage „Warum besuchst du die Universität?" wurden die IM (z.B. „Weil ich Freude daran habe neue Dinge zu erlernen.“) und EM (z.B. „Weil ich ohne Studium keinen hochbezahlten Job finden würde.") jeweils mit 12 Items gemessen. Schließlich wurde die AM mit vier 
Items (z. B. „Ehrlich gesagt, ich habe das Gefühl mit dem Studium meine Zeit zu verschwenden.") abgebildet (Koch, Schatz \& Borchert (in Druck); Vallerand et al., 1992). Aufgrund zunehmender Kritik an der Dimensionalität der AMS erfolgt eine Überprüfung der Faktorenstruktur der Skala mithilfe einer konfirmatorischen Faktorenanalyse (KFA) (z.B. Cokley, Bernard, Cunningham \& Motoike, 2001; Otis, Grouzet \& Pelletier, 2005). Diese stellt auf Basis der Selbstbestimmungstheorie sowie der bisherigen empirischen Befunde ein Ein-Faktor-Modell einem Drei-, einem Fünf- sowie einem Sieben-Faktoren-Modell gegenüber. Während das Ein-Faktor-Modell alle Items als einen Faktor abbildet, differenziert das Drei-Faktoren-Modell die Dimensionen EM, IM und AM. Das Fünf-FaktorenModell differenziert hinsichtlich der Subdimensionen externale $\mathrm{EM}$, introjizierte $\mathrm{EM}$, identifizierte $\mathrm{EM}$, IM und AM (Otis, Grouzet \& Pelletier, 2005). Demgegenüber untergliedert das Sieben-Faktoren-Modell zusätzlich zum Fünf-Faktoren-Modell die IM in drei Subdimensionen, IM Wissen, IM Kompetenz, IM Stimulation (Vallerand et al., 1992). Insgesamt wies das Fünf-Faktoren-Modell im Vergleich zu den übrigen Modellen den besten und insgesamt einen sehr guten Modellfit auf $(G F I=.93, C F I=.91$, $S R M R=.05, R M S E A=.06$, siehe Tabelle 1). Damit werden die Befunde u.a. von Otis, Grouzet \& Pelletier (2005) bestätigt und Abstand von einer weiteren Ausdifferenzierung der IM genommen. Folglich basiert die nachfolgende Analyse der AMS auf 20 Items, wobei jeweils vier Items eine Subdimension darstellen. In Einklang mit den Ergebnissen von Vallerand et al. (1992) bestätigen die Cronbachs Alpha-Werte der einzelnen Subdimensionen $\left(\alpha_{\text {externaleEM }}=\right.$ $\left..80, \alpha_{\text {introjizierteEM }}=.79, \alpha_{\text {identifizierteEM }}=.68, \alpha_{\mathrm{IM}}=.84, \alpha_{\mathrm{AM}}=.79\right)$ sowie der Gesamtskala $(\alpha=.74)$ die hohe Reliabilität der Skala. Die Interkorrelationen zwischen den Subdimensionen entsprechen den in der Literatur vorgeschlagenen Ausprägungen und liegen im Bereich von (-.522 bis .756; $p<.01)$.

Die Messungen der Persönlichkeitsmerkmale Gewissenhaftigkeit und Neurotizismus erfolgen mithilfe der FFI30-Skala nach Körner et al. (2008). Diese erfasst insgesamt fünf Persönlichkeitsmerkmale, welche sich inhaltlich auf das jeweilige Persönlichkeitsmerkmal beziehen (z.B. Pervin \& John, 1999). Die Antworten erfolgen auf einer fünfstufigen Likert-Skala $(1=$,trifft nicht zu“ bis $5=$, trifft völlig zu“). In diesem Beitrag liegt der Fokus zum einen auf der Gewissenhaftigkeit, welche mit sechs Items (z.B. "Ich halte meine Sachen generell ordentlich und sauber.") erfasst wurde, sowie zum anderen auf dem Neurotizismus. Dieser wurde ebenfalls mit sechs Items (z.B. „Ich fühle mich anderen oft unterlegen.") gemessen. Eine konfirmatorische Faktorenanalyse bestätigt den verbesserten Modellfit des Zwei-Faktoren-Modells $(G F I=.95, C F I=.92$, $S R M R=.06, R M S E A=.07$, siehe Tabelle 2). Der Cron- bachs Alpha-Wert für das Persönlichkeitsmerkmal Gewissenhaftigkeit liegt bei $\alpha=.74$, während hinsichtlich des Neurotizismus ein Cronbachs Alpha-Wert von $\alpha=.82$ erreicht wird. Dementsprechend werden die beiden Persönlichkeitsmerkmale Gewissenhaftigkeit und Neurotizismus nachfolgend analog zur theoretischen Herleitung in zwei getrennten Faktoren betrachtet.

Ferner werden verschiedene bildungsbezogene und demografische Angaben als Kontrollvariablen herangezogen. Es ist davon auszugehen, dass sich die Zusammenhänge zwischen den unabhängigen und abhängigen Variablen sowohl aufgrund der Anzahl der Fachsemester als auch auf Basis des Lernaufwandes unterscheiden (z.B. Brodhagen \& Gettinger, 2012; Vansteenkiste, Lens \& Deci, 2006). Des Weiteren legen empirische Befunde nahe, dass sich das Abitursystem (G8- oder G9-System) auf die Persönlichkeitsentwicklung und die akademische Motivation sowie auf die AL der Studenten auswirken (z.B. Homuth, 2017; Kristen, 2014). Um darüber hinaus Verzerrungen durch die besuchte Universität zu vermeiden, wird auch für diese Variable kontrolliert. Zudem hat die akademische Ausbildung der Eltern, als ein Indiz für den sozioökonomischen Status, einen erheblichen Einfluss auf die akademische Motivation und die AL der Studenten, sodass für diese Variable zu kontrollieren ist (in Anlehnung an Dennis, Phinney \& Chuateco, 2005; Fereidooni, 2012). Ein akademischer Bildungsstand der Eltern liegt vor, sofern mindestens ein Elternteil einen Abschluss an einer Hochschule erreicht hat. Des Weiteren werden das Geschlecht und das Alter der Studenten als Kontrollvariablen aufgenommen (z.B. Chee, Pino \& Smith, 2005; Vallerand et al., 1992). Gleichzeitig soll auch ein Einfluss der unterschiedlichen Erhebungszeitpunkte auf die Ergebnisse vermieden werden, weshalb hierfür ebenfalls kontrolliert wird.

\section{Datenauswertung}

Die Analyse von Leistungsunterschieden unter Berücksichtigung des Migrationshintergrundes erfolgt in drei Schritten. Dabei wird der Migrationshintergrund der Studenten stets als dichotome Gruppenvariable genutzt, um Unterschiede zwischen den beiden Gruppen „Student mit Migrationshintergrund“ und "Student ohne Migrationshintergrund" herauszustellen.

Im ersten Schritt erfolgt ein Überblick über die deskriptiven Ergebnisse. Im zweiten Schritt wird die Validität der Messmodelle der latenten Konstrukte im Rahmen von konfirmatorischen Faktorenanalysen analysiert. Schließlich werden im dritten Schritt die Hypothesen 1-5 geprüft. Die Schätzung des dazu entwickelten Strukturgleichungsmodells erfolgt in SPSS AMOS mithilfe der MaximumLikelihood-Methode. 
Tabelle 1. Gütekriterien zu den Messmodellen der akademischen Motivation

\begin{tabular}{lcccc}
\hline Modell & GFI & CFI & SRMR & RMSEA \\
\hline Ein-Faktor-Modell $^{1}$ & .575 & .466 & .133 & .133 \\
Drei-Faktoren-Modell $^{2}$ & .753 & .706 & .093 & .099 \\
Fünf-Faktoren-Modell $^{3}$ & .929 & .909 & .051 & .064 \\
Sieben-Faktoren-Modell $^{4}$ & .895 & .883 & .058 & .064 \\
\hline
\end{tabular}

Anmerkungen: ${ }^{1}$ Alle Items werden in einem Faktor abgebildet, ${ }^{2}$ Die intrinsische sowie extrinsische Motivation und die Amotivation werden jeweils als eigenständige Faktoren abgebildet, ${ }^{3}$ In Anlehnung an Otis et al. (2005) werden in diesem Modell nur 20 Items einbezogen, wobei die Subdimensionen intrinsische Motivation, externale extrinsische Motivation, introjizierte extrinsische Motivation, identifizierte extrinsische Motivation sowie die Amotivation jeweils als ein Faktor abgebildet werden, ${ }^{4} \mathrm{Die}$ Subdimensionen der intrinsischen Motivation (Wissen, Kompetenz, Stimulation) sowie diejenigen der extrinsischen Motivation (external, introjiziert und identifiziert) und die Amotivation werden als eigenständige Faktoren abgebildet.

Tabelle 2. Gütekriterien zu den Messmodellen der Big-Five Persönlichkeitsmerkmale

\begin{tabular}{lcccc}
\hline Modell & GFI & CFI & SRMR & RMSEA \\
\hline Ein-Faktor-Modell ${ }^{1}$ & .742 & .609 & .144 & .156 \\
Zwei-Faktoren-Modell $^{2}$ & .951 & .916 & .062 & .073 \\
\hline
\end{tabular}

Anmerkungen: ${ }^{1}$ Alle 12 Items werden in einem Faktor abgebildet, ${ }^{2}$ Die Items der Gewissenhaftigkeit und des Neurotizismus werden jeweils als eigenständige Faktoren abgebildet.

\section{Ergebnisse}

Die im ersten Schritt erzielten deskriptiven Befunde in Form von Mittelwerten, Standardabweichungen und Korrelationen sind Tabelle $3 \mathrm{zu}$ entnehmen. Im Hinblick auf das Selbstbestimmungskontinuum wurden hohe positive Korrelationen zwischen angrenzenden Subdimensionen der akademischen Motivation bestätigt. Die stärkste negative Korrelation wurde jedoch entgegen der theoretischen Überlegungen zwischen der Amotivation und der identifizierten EM erreicht (Fairchild, Horst, Finney \& Barron, 2005; Vallerand, Pelletier, Blais, Brière, Senecal \& Vallières, 1993). Zudem zeigen die Ergebnisse größtenteils signifikante Korrelationen zwischen den hergeleiteten Determinanten und der AL für beide Gruppen von Studenten. Auffällig ist, dass bei Studenten mit Migrationshintergrund alle Dimensionen der EM keine signifikanten Korrelationen mit der AL aufweisen. Darüber hinaus wurde für die Subdimension ,introjiziert" der EM eine signifikante negative Korrelation mit der AL von Studenten ohne Migrationshintergrund ermittelt. Dies lässt einen Widerspruch zu, dem bei beiden Gruppen von Studenten erwarteten positiven Zusammenhang zwischen der EM und der AL vermuten.

Im zweiten Schritt erfolgt die Analyse des gesamten zugrundeliegenden Messmodells. Im Rahmen einer KFA zeigte das Sieben-Faktoren-Modell den besten Fit zu den empirischen Daten $(G F I=.92, C F I=.90, S R M R=.05, R M S E A=.05)$.

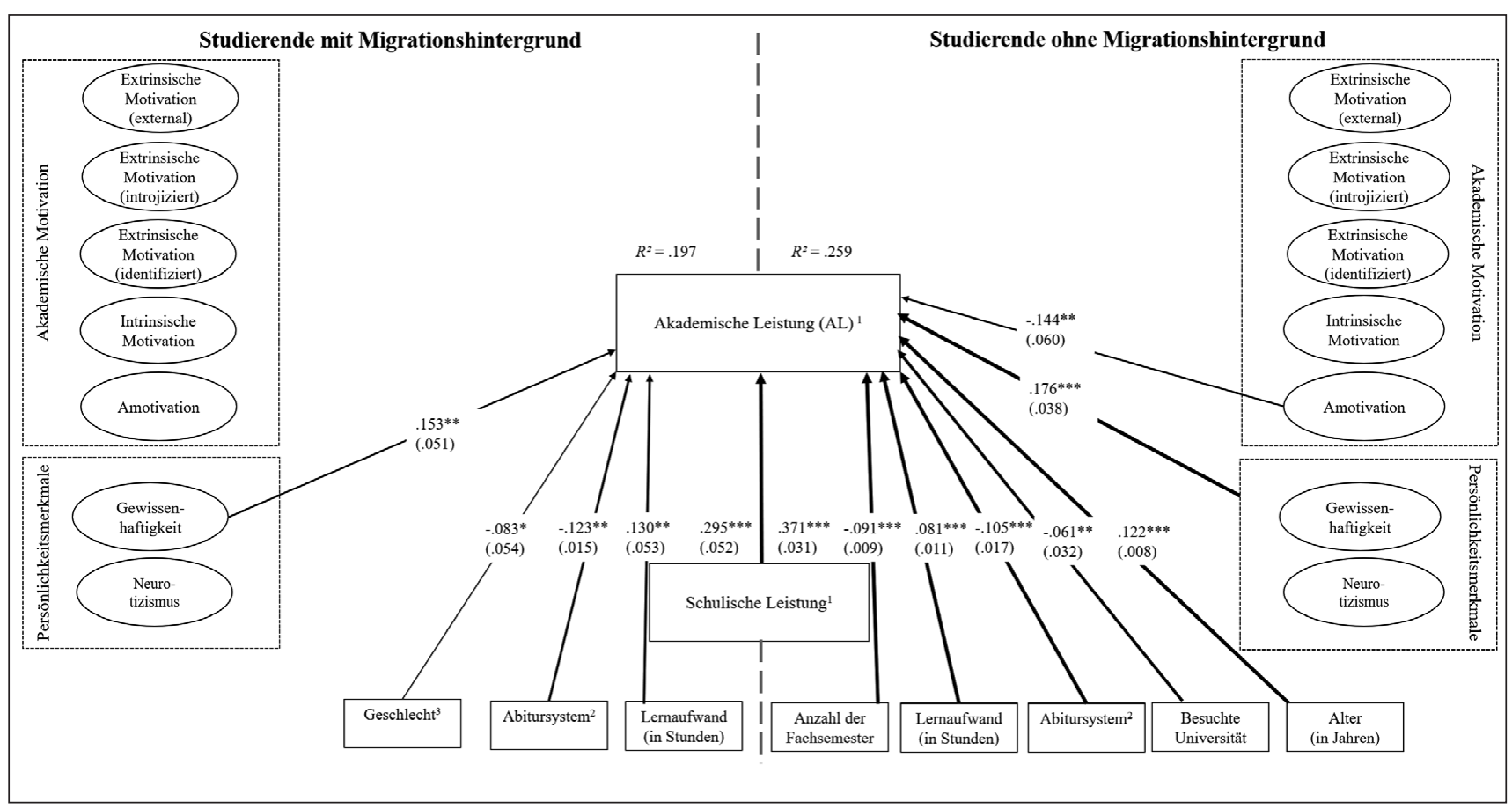

Anmerkungen: Angabe der statistisch signifikanten, standardisierten Pfadkoeffizienten sowie deren Standardfehler; Darstellung ohne Interkorrelationen zwischen den Prädiktoren. GFI $=.91, \mathrm{CFI}=.89, \mathrm{SRMR}=.04, \mathrm{RMSEA}=.03 ;{ }^{*} p<.10,{ }^{*} p<.05,{ }^{* *} p<.01 .{ }^{1}$ Variablenkodierung: $1=$ sehr gut bis $4=$ ausreichend; ${ }^{2}$ Variablenkodierung: 1 = G8-System (12 Jahre), 2 = G9-System (13 Jahre), ${ }^{3}$ Variablenkodierung: 0 = männlich, 1 = weiblich.

Abbildung 1. Determinanten der akademischen Leistung von Studenten mit und ohne Migrationshintergrund. 
Tabelle 3. Mittelwerte, Standardabweichungen und Interkorrelationen zwischen den relevanten Variablen

\begin{tabular}{|c|c|c|c|c|c|c|c|c|c|c|}
\hline \multirow[b]{2}{*}{ Variable } & \multicolumn{2}{|c|}{$\begin{array}{l}\text { Studenten mit } \\
\text { Migrations- } \\
\text { hintergrund }\end{array}$} & \multicolumn{2}{|c|}{$\begin{array}{l}\text { Studenten ohne } \\
\text { Migrations- } \\
\text { hintergrund }\end{array}$} & \multirow[b]{2}{*}{1} & \multirow[b]{2}{*}{2} & \multirow[b]{2}{*}{3} & \multirow[b]{2}{*}{4} & \multirow[b]{2}{*}{5} & \multirow[b]{2}{*}{6} \\
\hline & $M$ & $S D$ & $M$ & $S D$ & & & & & & \\
\hline 1 EM external & 4.031 & .750 & 3.943 & .740 & & $.385^{\star \star}$ & $.491 \star \star$ & .011 & $-.099 * \star$ & $.117 * *$ \\
\hline 2 EM introjiziert & 3.218 & .986 & 3.147 & .946 & $.372 * \star$ & & $.279 * \star$ & $.213^{* *}$ & -.001 & 0.032 \\
\hline 3 EM identifiziert & 3.959 & .708 & 3.870 & .692 & $.590 \star \star$ & $.326 * \star$ & & $.278 * \star$ & $-.367 * \star$ & $.192 * \star$ \\
\hline $4 \mathrm{IM}$ & 3.600 & .806 & 3.659 & .769 & $.141^{\star \star}$ & $.233^{\star \star}$ & $.384^{\star \star}$ & & $-.317 * *$ & $.191 * \star$ \\
\hline $5 \mathrm{AM}$ & 1.698 & .784 & 1.636 & .699 & $-.215^{\star \star}$ & $-.091 \star$ & $-.437 \star \star$ & $-.374^{\star \star}$ & & $-.330 * \star$ \\
\hline 6 Gewissenhaftigkeit & 3.909 & .662 & 4.008 & .641 & $.179 * \star$ & $.089 *$ & $.271 \star \star$ & $.259 * \star$ & $-.360 * \star$ & \\
\hline 7 Neurotizismus & 2.404 & .807 & 2.313 & .782 & .012 & $.146^{\star \star}$ & -.073 & $-.161 * \star$ & $.325^{\star \star}$ & $-.279 * \star$ \\
\hline 8 Akademische Leistung ${ }^{1}$ & 2.558 & .538 & 2.434 & .575 & .040 & -.017 & .057 & $.119 * *$ & $-.155^{\star \star}$ & $.214^{\star *}$ \\
\hline 9 Schulische Leistung 1 & 2.363 & .554 & 2.272 & .539 & .067 & -.043 & .062 & $.084^{\star}$ & -.049 & $.159 * *$ \\
\hline 10 Anzahl der Fachsemester & 4.000 & 1.998 & 3.906 & 1.847 & $-.113 \star \star$ & -.002 & $-.083 *$ & -.070 & $.097 \star$ & -.065 \\
\hline 11 Lernaufwand (in Stunden) & 2.723 & 1.463 & 2.654 & 1.448 & .022 & .009 & $.173^{\star \star}$ & $.178 * *$ & $-.107 * \star$ & $.181 * *$ \\
\hline 13 Abitursystem² & 1.839 & 1.765 & 1.487 & 1.034 & -.025 & .027 & .045 & .001 & -.059 & -.022 \\
\hline 14 Name der besuchten Universität $^{3}$ & 2.670 & 1.470 & 2.718 & 1.629 & -.056 & $.089 *$ & -.031 & .039 & $.148 * \star$ & -.001 \\
\hline 15 Akademische Ausbildung der Eltern ${ }^{4}$ & .351 & .478 & .474 & .500 & .043 & -.020 & .061 & $.082^{*}$ & -.003 & .001 \\
\hline 16 Geschlecht $^{5}$ & .557 & .497 & .475 & .500 & .009 & .017 & .074 & -.003 & -.054 & $.154^{\star *}$ \\
\hline 17 Alter (in Jahren) & 21.873 & 2.885 & 21.631 & 2.631 & $-.103 * \star$ & .072 & -.012 & $.081^{\star}$ & .002 & $-.092^{*}$ \\
\hline 18 Erhebungszeitpunkt $^{6}$ & 1.535 & .499 & 1.614 & .487 & -.034 & .035 & -.019 & .010 & .036 & .019 \\
\hline
\end{tabular}

In diesem werden sowohl die fünf Subdimensionen der akademischen Motivation (EM external, EM introjiziert, EM identifiziert, IM und AM) als auch die Gewissenhaftigkeit und der Neurotizismus als eigenständige Faktoren abgebildet. Dieses Ergebnis bestätigt die Konstrukt- und Diskriminanzvalidität, sodass die sieben genannten Faktoren im Folgenden getrennt voneinander untersucht werden. Daran anschließend erfolgt die Analyse des Strukturgleichungsmodells (SGM), um die aufgestellten Hypothesen zu den $\mathrm{Zu}-$ sammenhängen zwischen der Motivation sowie den Persönlichkeitsmerkmalen und der AL von Studenten mit und ohne Migrationshintergrund zu untersuchen (H1-5). Die Güte des SGMs weist einen guten Modellfit auf $(G F I=.91$, $C F I=.89, S R M R=.04, R M S E A=.03$ ). Abbildung 1 stellt die standardisierten Pfadkoeffizienten für Studenten mit und ohne Migrationshintergrund dar.

Um die Unterschiede zwischen Studenten mit und ohne Migrationshintergrund hinsichtlich der untersuchten Determinanten bewerten zu können, wird zunächst die relative Erklärungskraft der Determinanten für beide Gruppen miteinander verglichen. Hierbei zeigt sich, dass der Anteil der erklärten Varianz zwar unter den Studenten mit Migrationshintergrund mit 19,70\% $\left(R^{2}=.197\right)$ deutlich geringer ist im Vergleich zu Studenten ohne Migrationshintergrund $\left(R^{2}=.259\right)$. Dennoch kann für beide Gruppen von einer guten Modellanpassung gesprochen werden. Für
Studenten ohne Migrationshintergrund lässt sich demnach schließen, dass die unabhängigen Variablen (akademische Motivation, Gewissenhaftigkeit und Neurotizismus) besser geeignet sind, die Varianz in der $\mathrm{AL} \mathrm{zu}$ erklären, als für Studenten mit Migrationshintergrund.

Die Ergebnisse des SGMs bestätigen zunächst, dass für beide Gruppen von Studenten die schulische Leistung in einem positiven, statistisch signifikanten Zusammenhang zur AL steht. Hierbei erwies sich der genannte Zusammenhang für Studenten mit Migrationshintergrund $(\beta=.295$, $p<.01)$ als wenig schwächer im Vergleich zu Studenten ohne Migrationshintergrund $(\beta=.371, p<.01)$. In Bezug auf die akademische Motivation zeigen die empirischen Befunde, dass bei den Studenten mit Migrationshintergrund die akademische Motivation hinsichtlich keiner der Subdimensionen einen Zusammenhang zur AL aufweist. Entgegen der Hypothesen 1a-1c, wurden weder unter Studenten mit Migrationshintergrund noch unter Studenten ohne Migrationshintergrund statistisch signifikante Zusammenhänge zwischen den Subdimensionen der EM und der AL ermittelt. Trotz eines positiven Zusammenhangs zwischen der externalen EM und der AL wird Hypothese 1a angesichts der in beiden Gruppen fehlenden Signifikanz verworfen. Darüber hinaus sind die Zusammenhänge zwischen der introjizierten EM und der AL in beiden Gruppen negativ ausgeprägt und nicht signifikant, sodass Hypothe- 


\begin{tabular}{|c|c|c|c|c|c|c|c|c|c|c|}
\hline 7 & 8 & 9 & 10 & 11 & 12 & 13 & 14 & 15 & 16 & 17 \\
\hline $.058 *$ & .043 & .036 & $-.080 * \star$ & -.023 & $-.071 *$ & $.060 *$ & .018 & .021 & $-.160 * *$ & -.034 \\
\hline $.226 * \star$ & $-.063^{*}$ & $-.112^{\star *}$ & -.028 & .024 & -.016 & -.029 & -.030 & $.066^{\star}$ & .010 & $-.065^{\star}$ \\
\hline$-.070 *$ & $.119 * *$ & $.097 \star \star$ & $-.120 \star \star$ & .038 & $-.068 *$ & -.010 & $.077 * \star$ & $.069 *$ & $-.195 * \star$ & $-.056^{\star}$ \\
\hline$-.097 \star \star$ & $.155 * \star$ & $.096 * \star$ & $-.081 * \star$ & $.195^{\star \star}$ & .028 & .005 & $.080 * *$ & .054 & -.015 & -.029 \\
\hline $.330 * \star$ & $-.218^{\star \star}$ & -.030 & .052 & $-.111 * \star$ & .025 & $.073^{*}$ & .011 & $-.064^{\star}$ & .023 & -.005 \\
\hline \multirow[t]{2}{*}{$-.183^{\star \star}$} & $.261 \star \star$ & $.114^{\star *}$ & .036 & $.278 * \star$ & .019 & -.002 & -.003 & $.228 * \star$ & .023 & $.071 \star$ \\
\hline & $-.122 * \star$ & -.005 & .035 & .055 & -.007 & -.035 & -.029 & $.258 * \star$ & -.021 & -.032 \\
\hline$-.129 * \star$ & & $.383 * *$ & $-.077 \star \star$ & $.196 * *$ & $-.068 *$ & -.032 & $.080 * \star$ & $.074^{\star \star}$ & $-.085^{\star *}$ & -.025 \\
\hline-.016 & $.329 * \star$ & & $-.078 \star \star$ & $.125^{\star \star}$ & $-.080 \star \star$ & $.181^{\star \star}$ & $.161^{\star \star}$ & $.095^{\star \star}$ & $-.344^{* *}$ & -.032 \\
\hline $.081^{\star}$ & $-.086^{\star}$ & -.068 & & 0.023 & .043 & $-.099 * \star$ & -.040 & -.029 & $.369 * *$ & $.298 * *$ \\
\hline .031 & $.181 * \star$ & $.160 * \star$ & .033 & & .028 & $.077 * \star$ & .015 & $.157 \star \star$ & .032 & .044 \\
\hline-.002 & -.059 & $.190 * \star$ & .051 & $.147^{\star \star}$ & & -.034 & $-.135 * \star$ & -.045 & $.513 * \star$ & -.008 \\
\hline .011 & .066 & $.206 * \star$ & .004 & .049 & .056 & & $.109 * \star$ & $-.104^{\star \star}$ & -.035 & $.146 * \star$ \\
\hline .033 & .064 & $.164^{\star \star}$ & .022 & $.160 * *$ & .059 & $.094^{\star}$ & & -.007 & $-.183 * *$ & -.011 \\
\hline $.215^{\star *}$ & -.045 & $.111^{* *}$ & -.011 & .044 & .071 & $-.099 *$ & .024 & & $-.103 * \star$ & -.023 \\
\hline .007 & -.067 & $-.101 *$ & $.462 * *$ & $.109 * \star$ & $.291^{\star *}$ & $.084^{*}$ & -.0050 & -.044 & & $.086^{* *}$ \\
\hline-.001 & .059 & .039 & $.243 * \star$ & .073 & .005 & $.190 * *$ & .010 & -.019 & $.112^{\star \star *}$ & \\
\hline
\end{tabular}

Anmerkungen: $\mathrm{EM}=$ extrinsische Motivation, IM = intrinsische Motivation, $\mathrm{AM}=$ Amotivation. Die Korrelationen für Studenten mit Migrationshintergrund befinden sich unterhalb der Diagonalen ( $N=621)$, während sich die Korrelationen für Studenten ohne Migrationshintergrund oberhalb der Diagonalen befinden ( $N=1233) .{ }^{1}$ Variablenkodierung: 1 = sehr gut bis 4 = ausreichend; ${ }^{2}$ Variablenkodierung: 1 = G8-System (12 Jahre), 2 = G9-System (13 Jahre), ${ }^{3}$ Variablenkodierung: 1 = Universität Duisburg-Essen, 2 = Heinrich-Heine-Universität Düsseldorf, 3 = Bergische Universität Wuppertal, 4 = Ruhr-Universität Bochum, 5 = Technische Universität Dortmund, 6 = Universität zu Köln; $\quad{ }^{4}$ Variablenkodierung: $0=$ nein (nicht vorhaben), $1=$ ja (vorhanden); ${ }^{5}$ Variablenkodierung: 0 = männlich, 1 = weiblich, ${ }^{6}$ Variablenkodierung: 1 = Mai 2017, 2 = Mai 2018. ** $p<.01,{ }^{*} p<.05$ se $1 \mathrm{~b}$ abzulehnen ist. Gleichermaßen wurden entgegen Hypothese $1 \mathrm{c}$ negative, nicht signifikante Zusammenhänge zwischen der identifizierten EM und der AL identifiziert, sodass Hypothese 1c ebenfalls verworfen wird. Ferner wurden positive Zusammenhänge zwischen der IM und der AL festgestellt, welche jedoch in keiner der beiden Herkunftsgruppen statistisch signifikant sind, sodass Hypothese 2 nicht bestätigt wird. Hinsichtlich Hypothese 3 wurde in beiden Gruppen ein negativer Zusammenhang zwischen der AM und der AL identifiziert, welcher jedoch lediglich für Studenten ohne Migrationshintergrund statistisch signifikant ist $(\beta=-.144, p<.01)$. Da der vermutete Zusammenhang aufgrund fehlender Signifikanz hinsichtlich der Studenten mit Migrationshintergrund nicht verglichen werden kann, wird Hypothese 3 nicht bestätigt. Ferner bestätigen die empirischen Befunde zunächst in beiden Gruppen einen positiven Zusammenhang zwischen der Gewissenhaftigkeit und der AL. Dieser Zusammenhang ist für Studenten mit Migrationshintergrund $(\beta=.153, p<.05)$ geringer als für Studenten ohne Migrationshintergrund $(\beta=.176, p<.01)$, sodass Hypothese 4 abgelehnt wird. In Bezug auf den Zusammenhang zwischen dem Neurotizismus und der AL, welcher in Hypothese 5 vermutet wurde, ist in beiden Gruppen kein statistisch signifikanter Zusammenhang festzustellen. Dementsprechend wird H5 abgelehnt.

\section{Diskussion}

Der vorliegende Beitrag erweitert den bisherigen Forschungsstand zu den Determinanten der AL von Studenten durch eine differenzierte Betrachtung von Studenten mit und ohne Migrationshintergrund. Hinsichtlich der hergeleiteten Wirkungszusammenhänge zeigen sich relevante Unterschiede zwischen beiden Herkunftsgruppen. In Anlehnung an Brändle \& Lengfeld (2015) konnte der vorliegende Beitrag die Vermutung bestätigen, dass die schulischen Leistungen die AL von Studenten mit Migrationshintergrund in Deutschland determinieren und damit relevante Unterschiede in den AL von Studenten mit und ohne Migrationshintergrund begründen. Zunächst ist festzustellen, dass unter Studenten mit Migrationshintergrund sowohl die schulischen Leistungen als auch die AL im Durchschnitt schlechter sind als unter Studenten ohne Migrationshintergrund (s. Tabelle 3). Zudem zeigen unsere Ergebnisse, dass der Zusammenhang zwischen der schulischen Leistung und der AL bei Studenten mit Migrationshintergrund schwächer positiv ausgeprägt ist. Ursachen der akademischen Leistungsunterschiede zwischen diesen beiden Herkunftsgruppen stellen möglicherweise soziostrukturelle Faktoren, wie z.B. unterschiedliche Bildungshintergründe und soziale Herkünfte, dar (z.B. Bülow-Schramm, 2016; Ebert \& Heublein, 2017; Kristen, Reimer \& Kogan, 2008). 
Darüber hinaus zeigen die empirischen Befunde, dass die akademische Motivation keinen direkten Einflussfaktor der AL von Studenten mit Migrationshintergrund im Vergleich zu Studenten ohne Migrationshintergrund darstellt. Überraschenderweise haben weder die Subdimensionen der EM noch die IM oder die AM statistisch signifikante Zusammenhänge zur AL von Studenten mit Migrationshintergrund.

Im Hinblick auf die Gruppe der Studenten ohne Migrationshintergrund sind ebenfalls keine statistisch signifikanten Zusammenhänge zwischen den Subdimensionen der EM und der AL sowie zwischen der IM und der AL festzustellen. Jedoch steht die AM in einem negativen Zusammenhang zur AL unter Studenten ohne Migrationshintergrund. Im Hinblick auf die Hypothesen 1a-1c zeigen die empirischen Befunde folglich, dass die Subdimensionen der EM für keine der beiden Herkunftsgruppen in einem positiven Zusammenhang zur AL stehen. Insofern wirkt sich die Orientierung an externen Konsequenzen bzw. Belohnungen (z.B. der erwünschte soziale Aufstieg) nicht direkt auf die AL von Studenten mit und ohne Migrationshintergrund aus. Der in der Immigrant-Optimism-Hypothesis enthaltene Wunsch von Migranten, sich durch Bildung in die Aufnahmegesellschaft zu integrieren, führt zwar zu einer erhöhten Motivationsausprägung (s. Tabelle 3), aber nicht zu einer besseren AL unter Studenten mit Migrationshintergrund. Somit findet sich zunächst keine Bestätigung für die hier abgeleiteten Vermutungen. Ferner übt die Verinnerlichung von fremden Zielen (z.B. die elterlichen Werte und Erwartungen) keinen direkten Einfluss auf die AL von Studenten mit und ohne Migrationshintergrund aus. Die im Sinne des VOC-Ansatzes realisierten utilitaristischen Eltern-Kind-Beziehungen in Migrantenfamilien sind demnach nicht relevant für die AL. Im Hinblick auf die IM sind auch die hier insgesamt fehlenden signifikanten positiven Wirkungsbeziehungen zwischen der IM und AL in beiden Herkunftsgruppen überraschend. Im Einklang mit u.a. Fairchild et al. (2005) hat somit die reine Neugier, neues Wissen zu erlangen, keinen Einfluss auf die AL der Studenten. Dieser erstaunliche Befund wirft die Frage auf, ob z.B. die Studien- und Prüfungsbedingungen an deutschen Universitäten ursächlich dafür sind. Unübersichtliche und intransparente Strukturen in der akademischen Lehre wie beispielsweise im Aufbau und Verlauf des Studiums sowie hinsichtlich des Prüfungssystems sind in der Forschung allgemein belegt. Negative Rahmenbedingungen erhöhen die Belastungen und das Stresslevel für Studenten (Bargel, Ramm \& Multrus, 2012; Ramm, Multrus, Bargel \& Schmidt, 2014). Solche Gegebenheiten haben das Potenzial dafür, dass die vorhandene wissenschaftliche Neugierde, die durch die intrinsische Motivation zum Ausdruck kommt, nicht zu besseren AL beiträgt. Im Kontext der hier ermittelten Befunde ist somit von Interesse, ob und mit welchen spezifischen Bedingungen die Entstehung der intrinsischen Motivation von Studenten mit und ohne Migrationshintergrund geweckt werden kann, um Studienerfolge zu fördern. Hier sind in Zukunft weitere Studien - auch experimentelle Designs - wünschenswert, um zu entsprechenden Erklärungsansätzen zu kommen.

Schließlich zeigen die vorliegenden empirischen Befunde, dass die Amotivation unter Studenten ohne Migrationshintergrund zu einer Verschlechterung der AL führt. Wie vermutet, führt eine fehlende Motivation sowie das als gering wahrgenommene Potential im eigenen Handeln zu einer schlechteren AL im Vergleich zu motivierten Studenten, sodass die Annahmen des Überblicksmodells des motivierten Handelns Bestätigung finden (Heckhausen \& Heckhausen, 2018; Vallerand et al., 1992). Da dieser Zusammenhang für Studenten mit Migrationshintergrund jedoch nicht signifikant ist, wird Hypothese 3 nicht bestätigt. In zukünftigen Studien ist diesem Ergebnis verstärkte Aufmerksamkeit zu widmen. Dabei gilt es, weitergehende Erkenntnisse zur Entstehung, zur Aufrechterhaltung, zu den Wirkungen sowie zu den Möglichkeiten der Beseitigung von akademischer Amotivation im Kontext beider Herkunftsgruppen zu gewinnen.

Hinsichtlich der Persönlichkeitsmerkmale erwies sich die Gewissenhaftigkeit im Einklang mit bisherigen empirischen Befunden als ein Prädiktor der AL in beiden Herkunftsgruppen (z.B. Poropat, 2009). Die Ergebnisse zeigen darüber hinaus, dass der entsprechende Zusammenhang zwischen der Gewissenhaftigkeit und der AL für Studenten mit Migrationshintergrund schwächer positiv ausgeprägt ist als für Studenten ohne Migrationshintergrund. Die Hypothese 4 wird folglich abgelehnt. Diesbezüglich gilt es, weitergehende Erkenntnisse hinsichtlich der hemmenden Faktoren des Zusammenhangs zwischen Gewissenhaftigkeit und AL bei Studenten mit Migrationshintergrund $\mathrm{zu}$ gewinnen. Schließlich wurde in beiden Herkunftsgruppen kein statistisch signifikanter Zusammenhang zwischen dem Neurotizismus und der AL nachgewiesen. So wird Hypothese 5 nicht bestätigt.

Obgleich das finale SGM unter Berücksichtigung der genannten Kontrollvariablen einen wesentlichen Anteil der Varianz in AL von Studenten mit und ohne Migrationshintergrund erklären konnte (19,70\% und 25,90\%), wird ein erheblicher Teil der AL nicht erklärt. Hier besteht die Möglichkeit, dass weitere Variablen, z. B. der Umfang von Prüfungsvorbereitungen oder verschiedene Lernstrategien, in beiden Herkunftsgruppen eine Relevanz für die AL haben. Daher sind bezüglich solcher weiteren Variablen Nachfolgestudien wünschenswert. Darüber hinaus geben die erzielten Befunde Anlass für Nachfolgestudien, um das Ausmaß und die Relevanz von institutioneller Diskriminierung im Hinblick auf die Entstehung von akademischer Motivation und deren Wirkung auf die AL zu un- 
tersuchen. Institutionelle Diskriminierung kann nicht nur in der Gruppe der Studenten mit Migrationshintergrund aufgrund der ethnisch bedingten Herkunft empfunden werden, sondern auch unter Studenten ohne Migrationshintergrund, z.B. weil sie aus einem nicht akademischen Elternhaus stammen oder spezifische Handicaps aufweisen. Insofern ist die Forschung gefordert, den Entscheidungsträgern in Universitäten zukünftig weitere relevante Informationen hinsichtlich der Motivationsentstehung sowie den Unterstützungsbedarf von Studenten beider Gruppen zu liefern, um die AL von Studenten zu fördern (z.B. Morris-Lange \& Brands, 2015).

Insgesamt sind die Befunde dieser empirischen Studie jedoch aufgrund der Operationalisierung des Migrationshintergrundes der Studenten mit Vorsicht zu interpretieren. Migration ist ein vielschichtiges Phänomen, sodass stärkere Differenzierungen der Gruppe „Studenten mit Migrationshintergrund" weitergehende und spezifische Erkenntnisse liefern werden. Hier sind beispielsweise die individuelle Sozialisationshistorie, die kulturellen Hintergründe oder die dominierende Verkehrssprache innerhalb der Familie in zukünftigen Untersuchungen einzubeziehen (z. B. Jaksztat, 2014; Kemper, 2017). Darüber hinaus ist zu klären, inwiefern weitere Kontext- und Umweltfaktoren, wie soziostrukturelle Faktoren oder familiäre Ressourcen zur Erklärung von Unterschieden zwischen den Herkunftsgruppen relevant sind (Bülow-Schramm, 2016; Diehl, Hunkler \& Kristen, 2016; Kristen, Reimer \& Kogan, 2008). Ferner können der sozioökonomische Status, die familiäre Kultur oder die Unterstützung durch Eltern oder Lehrkräfte zumindest teilweise zur Erklärung von Bildungsbarrieren oder -ressourcen dienen (z.B. Becker, 2011; Dekker \& Fischer, 2008; Diefenbach, 2010; King \& McInerney, 2014; Kristen, Reimer \& Kogan, 2008). Auch wenn sich in den bisherigen Befunden hinsichtlich der Studienfachwahl nur geringfügig migrationsspezifische Unterschiede ausmachen lassen (z. B. Kristen, 2014), könnten die mit dem Studiengang einhergehenden erlebten Studien- und Prüfungsbedingungen als zusätzliche, externe Kontextfaktoren zur Erklärung von Leistungsunterschieden herangezogen werden. Dahingehend sind insbesondere wirtschaftswissenschaftliche Studiengänge von erheblicher Relevanz für die Untersuchung, da u.a. Wirtschaftswissenschaften in beiden Herkunftsgruppen ein gefragter Studiengang ist (Kristen, 2014).

Darüber hinaus limitiert die Operationalisierung der akademischen Motivation die ermittelten Ergebnisse. Dahingehend ist insbesondere die Dimensionalität der AMS kritisch zu diskutieren. Während die ursprünglich entwickelte Sieben-Faktoren-Struktur der akademischen Motivation durch unzureichende Reliabilitäten und mangelnde Modellgüte gekennzeichnet ist (Cokley et al., 2001; Fairchild et al., 2005), vernachlässigt die stattdessen empfohlene Fünf-Faktoren-Struktur die Subdimensionen
Kompetenz und Stimulation der IM (z. B. Otis, Grouzet \& Pelletier, 2005). Auf Basis des bisherigen Forschungsstands wurde hier die Fokussierung auf die Subdimension Wissen wegen hoher Interkorrelationen sowie nicht signifikanter Unterschiede zwischen den Subdimensionen der IM vorgenommen (Cokley et al., 2001; Fairchild et al., 2005). Diese Vorgehensweise steht im Einklang mit dem Selbstbestimmungskontinuum, welches eine einzige Subdimension der IM vorsieht. Diese Subdimension weist zudem die stärkste negative Korrelation mit der AM auf, sodass die IM und AM die Endpunkte des Kontinuums darstellen (Fairchild et al., 2005). Die hier ermittelten Befunde zeigen jedoch, dass die stärkste negative Korrelation zwischen der AM und der identifizierten EM vorliegt. Insgesamt zeigt sich somit, dass nicht nur die Dimensionalität der AMS und die Messung der IM weitergehend zu validieren sind. Darüber hinaus ist auch zu prüfen, ob und inwieweit die Annahme eines Selbstbestimmungskontinuums mit den beiden Extrempunkten der AM und der IM über ein entsprechendes Messinstrument aufrechterhalten werden kann. Ergänzend besteht zudem ein weiterer interessanter Zugang zur Modellierung motivational relevanter Faktoren der AL auf Basis der Selbstbestimmungstheorie darin, das Ausmaß der Befriedigung der drei zentralen Bedürfnisse Autonomie, Kompetenz und Zugehörigkeit zu operationalisieren (Ryan \& Deci, 2017). Diese Vorgehensweise bietet die Möglichkeit, diesbezüglich noch nicht vorhandene Befunde hinsichtlich der in diesem Beitrag betrachteten Herkunftsgruppen von Studenten herauszuarbeiten.

Darüber hinaus sind in Nachfolgestudien Längsschnittstudien wünschenswert. Insbesondere mithilfe motivationaler, persönlichkeitsbedingter sowie sozialpsychologischer Einflussgrößen in Kombination mit studienspezifischen Kontextfaktoren der AL besteht das große Potenzial, pädagogisch relevante Unterschiede zwischen Studenten mit und ohne Migrationshintergrund aufdecken zu können. Derartige Befunde ermöglichen schließlich evidenzbasierte Entscheidungen zur zukünftigen Gestaltung und Optimierung der Studienbedingungen bei heterogenen Studentenschaften.

\section{Literatur}

Areepattamannil, S. \& Freeman, J. G. (2008). Academic achievement, academic self-concept, and academic motivation of immigrant adolescents in the greater Toronto area secondary schools. Journal of Advanced Academics, 19 (4), 700 - 743.

Bargel, T., Ramm, M. \& Multrus, F. (2012). Schwierigkeiten und Belastungen im Bachelorstudium-wie berechtigt sind die studentischen Klagen. Beiträge zur Hochschulforschung, 34 (1), 26 - 41.

Becker, R. (Hrsg.). (2011). Integration durch Bildung. Wiesbaden: VS. 
Bienfait, A. (2015). Studienabbrecherinnen und -abbrecher mit Migrationshintergrund. Migration und Soziale Arbeit, 37 (2), $133-139$.

Brändle, T. \& Lengfeld, H. (2015). Erzielen Studierende ohne Abitur geringeren Studienerfolg? Zeitschrift für Soziologie, 44 (6), $447-472$.

Brodhagen, E. M. \& Gettinger, M. (2012). Academic learning time. In N. M. Seel (Hrsg.). Encyclopedia of the sciences of learning (S. 33 -36). New York: Springer.

Bülow-Schramm, M. (2016). Durchlässigkeit als Zielmarke für Übergänge im Hochschulsystem? In A. Lange-Vester \& T. Sander (Hrsg.). Soziale Ungleichheiten, Milieus und Habitus im Hochschulstudium (S. 269 - 286). Weinheim: Beltz Juventa.

Cano, M. Á., Castillo, L. G., Davis, M. J., López-Arenas, A., Vaquero, J., Thompson, K. V. et al. (2012). Dynamics of acculturation, enculturation, and ethnic identity: Influence of psychocultural constructs on conscientiousness and expectations of higher education among Latinos. International Journal for the Advancement of Counselling, 34 (3), $231-241$.

Carnein, M. \& Baykara-Krumme, H. (2013). Einstellungen zur familialen Solidarität im Alter: Eine vergleichende Analyse mit türkischen Migranten und Deutschen. Zeitschrift für Familienforschung, 25 (1), $29-52$.

Chamorro-Premuzic, T. \& Furnham, A. (2003). Personality traits and academic examination performance. European Journal of Personality, 17 (3), $237-250$.

Chee, K. H., Pino, N. W. \& Smith, W. L. (2005). Gender differences in the academic ethic and academic achievement. College Student Journal, 39 (3), 604-618.

Cokley, K. O., Bernard, N., Cunningham, D. \& Motoike, J. (2001). A psychometric investigation of the academic motivation scale using a United States sample. Measurement and evaluation in Counseling and development, 34 (2), $109-119$.

Costa, P. T. \& McCrae, R. R. (1992). Four ways five factors are basic. Personality and Individual Differences, 13 (6), 653 - 665.

Deci, E. L., Vallerand, R. J., Pelletier, L. G. \& Ryan, R. M. (1991). Motivation and education: The self-determination perspective. Educational Psychologist, 26 (3-4), 325 - 346.

Dekker, S. \& Fischer, R. (2008). Cultural Differences in Academic Motivation Goals: A Meta-Analysis Across 13 Societies. The Journal of Educational Research, 102 (2), 99 - 110.

Dennis, J. M., Phinney, J. S. \& Chuateco, L. I. (2005). The role of motivation, parental support, and peer support in the academic success of ethnic minority first-generation college students. Journal of College Student Development, 46 (3), 223 - 236.

Diefenbach, H. (2010). Kinder und Jugendliche aus Migrantenfamilien im deutschen Bildungssystem. Wiesbaden: Springer.

Diehl, C., Hunkler, C. \& Kristen, C. (2016). Ethnische Ungleichheiten im Bildungsverlauf. Eine Einführung. In C. Diehl, C. Hunkler \& C. Kristen (Hrsg.). Ethnische Ungleichheiten im Bildungsverlauf (S. 3-31). Wiesbaden: Springer.

Ebert, J. \& Heublein, U. (2017). Studienabbruch bei Studierenden mit Migrationshintergrund. Essen: Stiftung Mercator.

El-Mafaalani, A., Waleciak, J. \& Weitzel, G. (2017). Tatsächliche, messbare und subjektiv wahrgenommene Diskriminierung. In A. Scherr, A. El-Mafaalani \& G. Yüksel (Hrsg.). Handbuch Diskriminierung (S.173-189). Wiesbaden: Springer.

Fairchild, A. J., Horst, S. J., Finney, S. J. \& Barron, K. E. (2005). Evaluating existing and new validity evidence for the Academic Motivation Scale. Contemporary Educational Psychology, 30 (3), $331-358$.

Fereidooni, K. (2012). Kinder mit Migrationshintergrund im deutschen Schulwesen-Benachteiligung aus (Bildungs-) politischen Ursachen. Gesellschaft-Wirtschaft-Politik, 61 (3), $363-371$.
Goodman, S., Jaffer, T., Keresztesi, M., Mamdani, F., Mokgatle, D., Musariri, M. et al. (2011). An investigation of the relationship between students' motivation and academic performance as mediated by effort. South African Journal of Psychology, 41 (3), $373-385$

Hasse, R. \& Schmidt, L. (2012). Institutionelle Diskriminierung. In U. Bauer, U. H. Bittlingmayer \& A. Scherr (Hrsg.). Handbuch Bildungsund Erziehungssoziologie (S. 883 - 899). Wiesbaden: Springer.

Heckhausen, J. \& Heckhausen, H. (Hrsg.). (2018). Motivation und Handeln. Berlin: Springer.

Higgins, D. M., Peterson, J. B., Pihl, R. O. \& Lee, A. G. (2007). Prefrontal cognitive ability, intelligence, Big Five personality, and the prediction of advanced academic and workplace performance. Journal of Personality and Social Psychology, 93 (2), 298 - 319.

Hillebrecht, L. (2019). Studienerfolg von berufsbegleitend Studierenden. Dissertation. Wiesbaden: Springer.

Homuth, C. (2017). Die G8-Reform in Deutschland. Bamberg: Springer.

Igler, J., Ohle-Peters, A. \& McElvany, N. (2019). Mit den Augen eines Grundschulkindes. Zeitschrift für Pädagogische Psychologie, 33 (3-4), $191-205$.

Jaksztat, S. (2014). Bildungsherkunft und Promotionen. Zeitschrift für Soziologie, 43 (4), 286 - 301.

Jancz, M. W. (2000). Social and psychological adjustments of first generation Polish immigrants to Australia. Dissertation. Sydney: University of Sydney.

Kao, G. \& Tienda, M. (1995). Optimism and achievement: The educational performance of immigrant youth. Social Science Quarterly, 76 (1), $1-19$.

Kemper, T. (2017). Die schulstatistische Erfassung des Migrationshintergrundes in Deutschland. Journal for Educational Research Online, 9 (1), 144-168.

King, R. B. \& Mclnerney, D. M. (2014). Culture's Consequences on Student Motivation: Capturing Cross-Cultural Universality and Variability Through Personal Investment Theory. Educational Psychologist, 49 (3), $175-198$.

Koch, E., Schatz, S. \& Borchert, M. (in Druck). Motivation, motiviertes Handeln und Leistungsniveaus zukünftiger Hochschulabsolventen. Unveröffentlichtes Diskussionspapier. Duisburg: Universität Duisburg-Essen, $1-56$.

Komarraju, M., Karau, S. J. \& Schmeck, R. R. (2009). Role of the Big Five personality traits in predicting college students' academic motivation and achievement. Learning and Individual Differences, 19 (1), $47-52$.

Körner, A., Geyer, M., Roth, M., Drapeau, M., Schmutzer, G., Albani, C. et al. (2008). Persönlichkeitsdiagnostik mit dem NEO-FünfFaktoren-Inventar: Die 30-Item-Kurzversion (NEO-FFI-30). Psychotherapie. Psychosomatik. Medizinische Psychologie, 58 (06), $238-245$.

Kristen, C. (2014). Migrationsspezifische Ungleichheiten im deutschen Hochschulbereich. Journal for Educational Research Online, 6 (2), $113-134$.

Kristen, C. (2016). Migrationsspezifische Ungleichheiten im deutschen Hochschulbereich. In C. Diehl, C. Hunkler \& C. Kristen (Hrsg.). Ethnische Ungleichheiten im Bildungsverlauf (S. 113 - 134). Wiesbaden: Springer.

Kristen, C., Reimer, D. \& Kogan, I. (2008). Higher education entry of Turkish immigrant youth in Germany. International Journal of Comparative Sociology, 49 (2-3), 127-151.

McCrae, R. R., Costa Jr, P.T., Ostendorf, F., Angleitner, A., Hřebíčková, M., Avia, M. D. et al. (2000). Nature over Nurture: Temperament, Personality, and Life Span Development. Journal of Personality and Social Psychology, 78 (1), 173-186.

Moffitt, U., Juang, L. P. \& Syed, M. (2019). "We don't do that in Germany!" A critical race theory examination of Turkish heritage young adults' school experiences. Ethnicities, 19 (5), 830 - 857. 
Morris-Lange, S. \& Brands, F. (2015). Zugangstor Hochschule. Studie des SVR-Forschungsbereichs. Berlin.

Nauck, B. (2000). Eltern-Kind-Beziehungen in Migrantenfamilien. In Sachverständigenkommission 6. Familienbericht (Hrsg.). Familien ausländischer Herkunft in Deutschland (S.347-392). Wiebaden: Springer.

Nauck, B. (2001). Der Wert von Kindern für ihre Eltern. Kölner Zeitschrift für Soziologie und Sozialpsychologie, 53 (3), 407 - 435.

OECD. (2016). PISA 2015.

Olbermann, E. (2003). Soziale Netzwerke, Alter und Migration. Dissertation. Dortmund: Universität Dortmund.

Otis, N., Grouzet, F. M. \& Pelletier, L. G. (2005). Latent motivational change in an academic Setting: A 3-Year longitudinal study. Journal of Educational Psychology, 97 (2), 170 - 183.

Pervin, L. A. \& John, O. P. (1999). Handbook of personality. (2nd ed.). New York: The Guilford Press.

Poropat, A. E. (2009). A meta-analysis of the five-factor model of personality and academic performance. Psychological Bulletin, 135 (2), $1-68$.

Raiser, U. (2007). Erfolgreiche Migranten im deutschen Bildungssystem - es gibt sie doch. Münster: LIT-Verlag.

Ramm, M., Multrus, F., Bargel, T. \& Schmidt, M. (2014). Studiensituation und studentische Orientierungen: 12. Studierendensurvey an Universitäten und Fachhochschulen. Universität Konstanz.

Richardson, M., Abraham, C. \& Bond, R. (2012). Psychological correlates of university students' academic performance: A systematic review and meta-analysis. Psychological Bulletin, 138 (2), $353-387$.

Röbken, H. \& Mertens, A. (2013). Studienmotivation von Studierenden in heterogenen Lebenslagen. In A. Hanft \& K. Brinkmann (Hrsg.). Offene Hochschulen (S. 42 - 52). Münster: Waxmann.

Ryan, R. M. \& Deci, E. L. (2000a). Intrinsic and extrinsic motivations: Classic definitions and new directions. Contemporary Educational Psychology, 25 (1), $54-67$.

Ryan, R. M. \& Deci, E. L. (2000b). Self-determination theory and the facilitation of intrinsic motivation, social development, and well-being. American Psychologist, 55 (1), 68 - 78.

Ryan, R. M. \& Deci, E. L. (2017). Self-determination theory: Basic psychological needs in motivation, development, and wellness. New York: Guilford Publications.

Sartawi, A., Alsawaie, O. N., Dodeen, H., Tibi, S. \& Alghazo, I. M. (2012). Predicting Mathematics Achievement by Motivation and Self-Efficacy Across Gender and Achievement Levels. Interdisciplinary Journal of Teaching and Learning, 2 (2), 59-77.

Sprung, A. (2017). Dealing with diversity and anti-racism in adult education: An institutional perspective. Andragoška spoznanja, 23 (2), $39-50$.

Statistisches Bundesamt. (2019). Bevölkerung und Erwerbstätigkeit. Bevölkerung mit Migrationshintergrund. Ergebnisse des Mikrozensus 2018.
Tepecik, E. (2011). Bildungserfolge mit Migrationshintergrund: Biographien bildungserfolgreicher MigrantInnen türkischer Herkunft. (1. Aufl.). Wiesbaden: Springer.

Tjaden, J. D. \& Hunkler, C. (2017). The optimism trap: Migrants' educational choices in stratified education systems. Social Science Research, 67, 213-228.

Turner, E. A., Chandler, M. \& Heffer, R. W. (2009). The influence of parenting styles, achievement motivation, and self-efficacy on academic performance in college students. Journal of College Student Development, 50 (3), 337 - 346.

Vallerand, R. J., Pelletier, L. G., Blais, M. R., Briere, N. M., Senecal, C. \& Vallieres, E. F. (1992). The Academic Motivation Scale. Educational and Psychological Measurement, 52 (4), 1003 - 1017.

Vallerand, R. J., Pelletier, L. G., Blais, M. R., Brière, N. M., Senecal, C. \& Vallières, É. F. (1993). On the assessment of intrinsic, extrinsic, and amotivation in education. Educational and Psychological Measurement, 53 (1), $159-172$.

Vansteenkiste, M., Lens, W. \& Deci, E. L. (2006). Intrinsic Versus Extrinsic Goal Contents in Self-Determination Theory: Another Look at the Quality of Academic Motivation. Educational Psychologist, 41 (1), $19-31$.

Wach, F., Karbach, J., Ruffing, S., Brünken, R. \& Spinath, F. M. (2016). University students' satisfaction with their academic studies: Personality and motivation matter. Frontiers in Psychology, 7 (55), $1-12$.

\section{Historie}

Manuskript eingereicht: 07.03.2020

Manuskript nach Revision angenommen: 18.01.2021

Onlineveröffentlichung: 17.02.2021

\section{Förderung}

Open Access-Veröffentlichung ermöglicht durch die Universität Duisburg-Essen.

\section{ORCID}

Mona Höyng

(D) https://orcid.org/0000-0001-9734-515X

\section{Sc. Mona Höyng}

Universität Duisburg-Essen

Mercator School of Management

Lotharstraße 65

47057 Duisburg

Germany

mona.hoeyng@uni-due.de 